\section{Acute Unilateral Sacroiliitis Mimicking Infection on Magnetic Resonance Imaging with Response to Nonsteroidal Antiinflammatory Drugs: A Distinct Presentation of Spondyloarthritis?}

\section{To the Editor:}

Sacroiliitis is associated with the spondyloarthropathies ( $\mathrm{SpA}$ ) including ankylosing spondylitis, psoriatic arthritis, and reactive arthritis (ReA), and may be visualized using magnetic resonance imaging (MRI). Here, we describe 4 cases of acute unilateral sacroiliitis with florid MRI appearances that mimicked infection but demonstrated a prompt and complete response to nonsteroidal antiinflammatory drugs (NSAID).

All patients in this report have given written consent for publication. Institutional review board approval for ethics was not required because patients were managed according to generally accepted standards of care prior to this case series report.

All subjects were HLA-B27-negative males and presented with a rapid symptom onset of unilateral sacroiliitis lasting from 2 days to 4 weeks (Table 1). One subject had a prior history of ulcerative colitis (Case 2) in remission and 1 had scalp psoriasis (Case 3). There were prodromal symptoms in 2 subjects (Cases 1 and 4) with short-lived fever at presentation. Case 4 had a sore throat preceding the presentation with neutrophilia $\left(12.4 \times 10^{9} / 1\right)$, which prompted an infection screen. All 4 patients demonstrated a significant elevation in acute-phase markers, with a mean serum C-reactive protein (CRP) of $115 \mathrm{mg} / \mathrm{l}$. There were no overt clinical features of systemic inflammatory response.
Baseline MRI demonstrated florid bone marrow edema (BME) in 3 cases (Cases 1,2, and 4) affecting $>75 \%$ of the sacroiliac joints (SIJ) and moderate (affecting 25-75\%) in Case 3 (Figure 1). High signal was noted in surrounding muscle and soft tissue in all cases by the reporting radiologists, who observed the need to exclude infection. SIJ aspiration/biopsy was considered in all cases but not conducted because of the prompt symptom response following NSAID, with improvement in clinical variables and negative septic screen.

Case 2 was advised to continue empirical combined oral antibiotics for 4 weeks. In addition, he continued NSAID therapy for 8 weeks until complete symptom resolution. Case 4 cultured group A Streptococcus from a throat swab and borderline antistreptolysin titer of $466 \mathrm{IU} / \mathrm{ml}$ and 406 $\mathrm{IU} / \mathrm{ml}$, respectively, suggesting plausible post-streptococcal ReA. Repeat MRI was performed in 3 patients at a mean followup of 5 weeks, which demonstrated improved but persistent inflammatory changes. Further imaging thereafter revealed significant improvement in BME changes in Cases 1 and 3, at 2 and 5 months, respectively.

Sacroiliitis typifies SpA but can also occur in sepsis where diffuse soft tissue edema, in addition to BME, is characteristic ${ }^{1}$. The symptom onset in SpA can be acute and may include fever and raised CRP, therefore mimicking infection. Bilateral sacroiliitis is invariably inflammatory; however, an acute unilateral presentation is frequently reported in the literature as pyogenic or suspicious for atypical organisms ${ }^{1}$. The current case series demonstrates that acute unilateral sacroiliitis with "extreme" MRI appearances, particularly with extensive sacroiliac BME and adjacent periarticular muscle/soft tissue edema can, despite resembling infection,

Table 1. Clinical characteristics of 4 HLA-B27-negative subjects presenting with acute unilateral sacroiliitis.

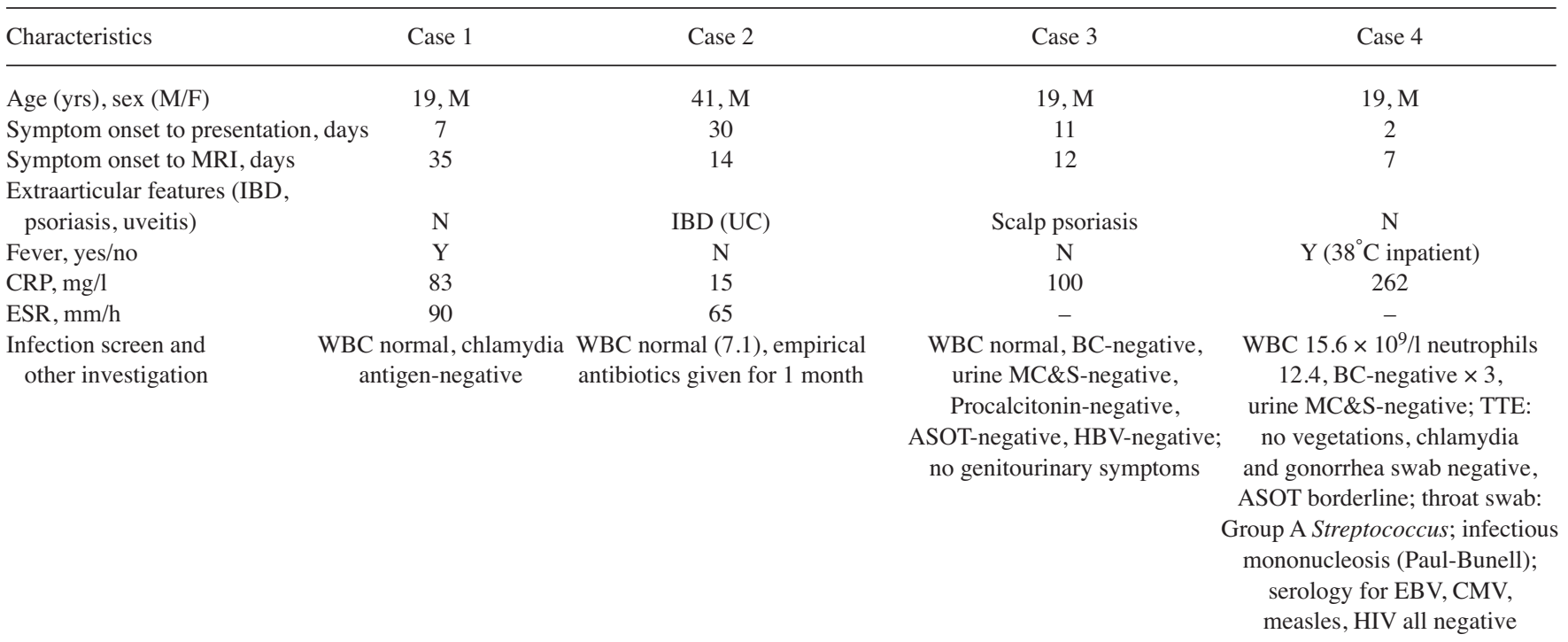

NSAID

Initial pain response from

NSAID, days

NSAID commencement to complete

resolution of symptoms, weeks

Symptom onset to complete

resolution*, weeks
Naproxen, $500 \mathrm{mg}$ bid

(diclofenac $75 \mathrm{mg}$ bid for

initial 2 weeks)
Etoricoxib, $90 \mathrm{mg}$ qd

Ibuprofen, $400 \mathrm{mg}$ tid
Etoricoxib, $120 \mathrm{mg} \mathrm{qd}$

* Complete resolution refers to disappearance of symptoms and substantial CRP improvement or normalization. IBD: inflammatory bowel disease; UC: ulcerative colitis; CRP: C-reactive protein; ESR: erythrocyte sedimentation rate; WBC: white blood cells; BC: blood cultures; MC\&S: microscopy culture and sensitivity; TTE: transthoracic echocardiogram; ASOT: antistreptolysin O titer; EBV: Epstein-Barr virus; CMV: cytomegalovirus; HBV: hepatitis B virus; HIV: human immunodeficiency virus; qd: once daily; bid: twice daily; tid: 3 times daily; MRI: magnetic resonance imaging; NSAID: nonsteroidal antiinflammatory drugs.

Personal non-commercial use only. The Journal of Rheumatology Copyright @ 2018 . All rights reserved. 
1

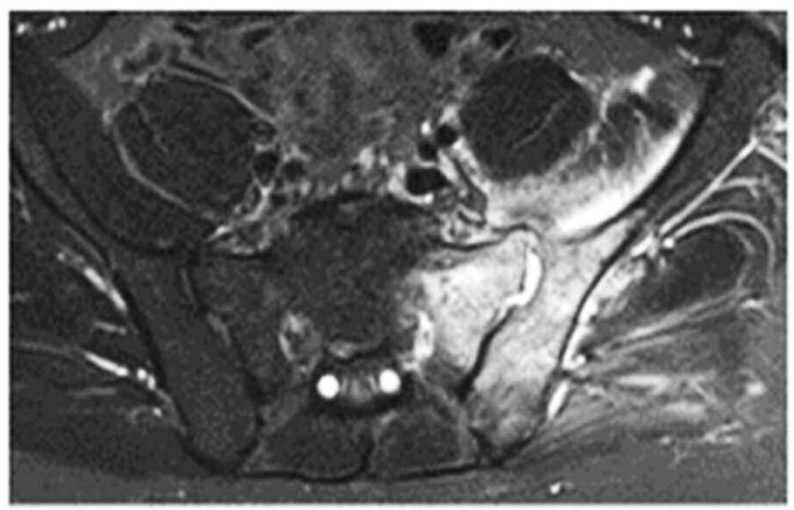

3

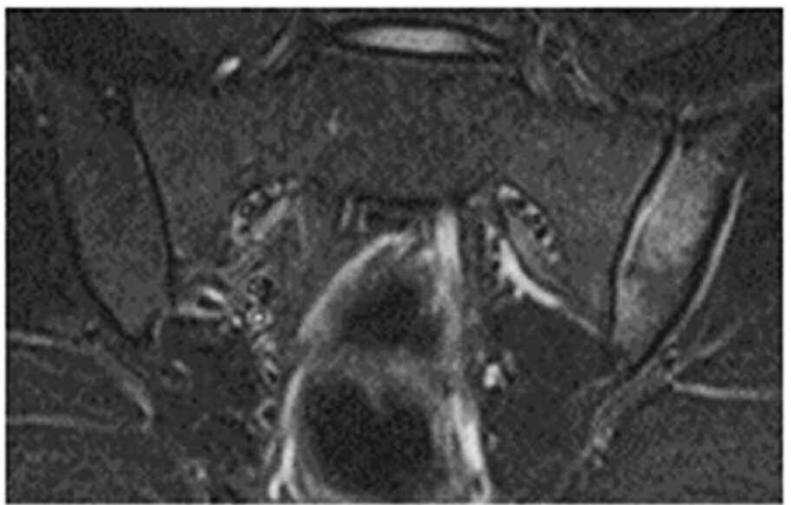

2

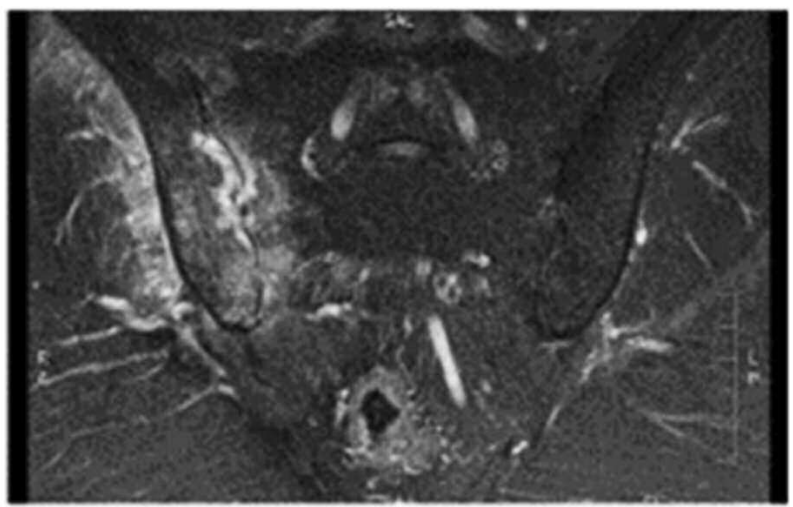

4

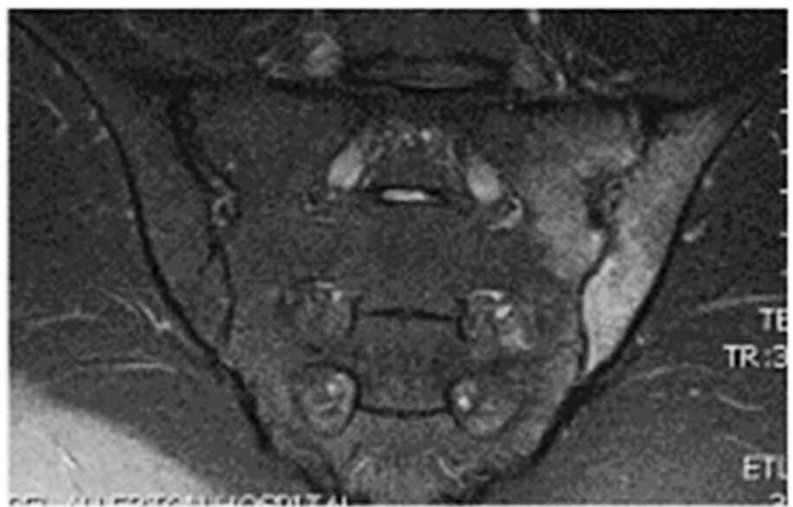

Figure 1. Coronal STIR MRI examination of the sacroiliac joints per case. Cases labeled by corresponding number. STIR: short-tau inversion recovery; MRI: magnetic resonance imaging.

represent a reactive process suggestive of an inflammatory $\mathrm{SpA}$. These cases illustrate the diagnostic challenge of differentiating infection versus inflammation. This is particularly important given that patients typically present through urgent appointments (Cases 3 and 4 presented to the emergency department requiring hospitalization). All patients demonstrated a good response to NSAID. Although the dose and duration of NSAID required to alter BME is unclear, our data support previous reports in the literature ${ }^{2}$. We acknowledge that the effect of NSAID cannot be quantitatively measured from these series, particularly as postinflammatory changes were still visible in 2 cases after 5 weeks. Remarkably, however, all patients were symptomfree within 8 weeks.

Acute unilateral sacroiliitis can be a manifestation of $\operatorname{ReA}^{3,4}$. During a Campylobacter jejuni outbreak, 1 in 15 cases of ReA presented with sacroiliitis ${ }^{4}$. Similarly, sacroiliitis is a rare manifestation of post-streptococcal $\operatorname{Re}^{5}$. Pseudosepsis has been observed in psoriasis, palmoplantar pustulosis, acne, and the synovitis, acne, pustulosis, hyperostosis, and osteitis syndrome but is an unusual cause for de novo acute unilateral sacroiliitis ${ }^{6}$. The severity of sacroiliitis at baseline regardless of HLA-B27 status has been shown to be a predictor of poor prognosis for radiographic progression, but little is known specifically for acute $\operatorname{ReA}^{7}$. When managing such cases, it is essential not to overlook infectious sacroiliitis typified on MRI by periarticular muscle edema, although the cases presented here also demonstrate that inflammatory disease can mimic such appearances ${ }^{8}$. Interestingly and although within the spectrum of SpA, our cases could not be classified according to the Assessment of Spondyloarthritis international Society classification criteria given the acute onset of symptoms of $<3$ months' duration $^{9,10}$.

These case reports highlight that significant reactive inflammatory sacroiliitis can yield MRI appearances mimicking infection. Thorough investigation should always be prioritized, but NSAID alone can be effective in resolving symptoms over several weeks, with eventual patient recovery.

\section{ACKNOWLEDGMENT}

The authors thank the patients for allowing their information to be shared for this report. This research is supported by the National Institute for Health Research (NIHR) Leeds Biomedical Research Centre. The views expressed are those of the authors and not necessarily those of the National Health Service (NHS), the NIHR, or the Department of Health, UK

SAYAM DUBASH ${ }^{\circledR}$, MBChB, FRCP, NIHR Leeds Biomedical Research Centre, Leeds Teaching Hospitals NHS Trust, and Leeds Institute of Rheumatic and Musculoskeletal Medicine, University of Leeds, Leeds, UK; COLIN PEASE, MD, FRCP, NIHR Leeds Biomedical Research Centre, Leeds Teaching Hospitals NHS Trust, and Leeds Institute of Rheumatic and Musculoskeletal Medicine, University of Leeds; AAMIR ASLAM, MRCP, DPhil, NIHR Leeds Biomedical Research Centre, Leeds Teaching Hospitals NHS Trust, and Leeds Institute of Rheumatic and Musculoskeletal Medicine, University of Leeds; DAVID COADY, MBBS, MRCP, MD, Rheumatology Department, Sunderland Royal Hospital, Sunderland; DENNIS McGONAGLE, FRCPI, PhD, NIHR Leeds Biomedical Research Centre, Leeds Teaching Hospitals NHS Trust, and Leeds Institute of Rheumatic and Musculoskeletal Medicine, University of Leeds; HELENA MARZO-ORTEGA, MRCP, PhD, NIHR Leeds Biomedical Research Centre, Leeds Teaching Hospitals NHS Trust, and Leeds Institute of Rheumatic and Musculoskeletal Medicine, University of Leeds, Leeds, UK. Address correspondence to Dr. H. Marzo-Ortega, LIRMM, Second floor, Chapel Allerton Hospital, Chapeltown Road, Leeds LS7 4SA, UK. E-mail: medhmo@leeds.ac.uk

\section{REFERENCES}

1. Slobodin G, Rimar D, Boulman N, Kaly L, Rozenbaum M, Rosner I, et al. Acute sacroiliitis. Clin Rheumatol 2016;35:851-6.

2. Varkas G, Jans L, Cypers H, Van Praet L, Carron P, Elewaut D, et al.

Personal non-commercial use only. The Journal of Rheumatology Copyright (c) 2018. All rights reserved. 
Brief report: Six-week treatment of axial spondyloarthritis patients with an optimal dose of nonsteroidal antiinflammatory drugs: early response to treatment in signal intensity on magnetic resonance imaging of the sacroiliac joints. Arthritis Rheumatol 2016;68:672-8.

3. Oates JK, Young AC. Sacro-iliitis in Reiter's disease. Br Med J 1959;1:1013-5.

4. Hannu T, Kauppi M, Tuomala M, Laaksonen I, Klemets P, Kuusi M. Reactive arthritis following an outbreak of Campylobacter jejuni infection. J Rheumatol 2004;31:528-30.

5. Mackie SL, Keat A. Poststreptococcal reactive arthritis: what is it and how do we know? Rheumatology 2004;43:949-54.

6. Rohekar G, Inman RD. Conundrums in nosology: synovitis, acne, pustulosis, hyperostosis, and osteitis syndrome and spondyloarthritis. Arthritis Rheum 2006;55:665-9.

7. Hannu T, Inman R, Granfors K, Leirisalo-Repo M. Reactive arthritis or post-infectious arthritis? Best Pract Res Clin Rheumatol 2006;20:419-33.
8. Kang Y, Hong SH, Kim JY, Yoo HJ, Choi JY, Yi M, et al. Unilateral sacroiliitis: differential diagnosis between infectious sacroiliitis and spondyloarthritis based on MRI findings. AJR Am J Roentgenol 2015;205:1048-55

9. Bennett AN, Marzo-Ortega H, Emery P, McGonagle D; Leeds Spondyloarthropathy Group. Diagnosing axial spondyloarthropathy. The new Assessment in SpondyloArthritis international Society criteria: MRI entering centre stage. Ann Rheum Dis 2009;68:765-7.

10. van der Heijde D, Ramiro S, Landewé R, Baraliakos X, Van den Bosch F, Sepriano A, et al. 2016 update of the ASAS-EULAR management recommendations for axial spondyloarthritis. Ann Rheum Dis 2017;76:978-91.

J Rheumatol First Release August 15, 2018; doi:10.3899/jrheum.171456 